\title{
Lignans isolated from the ethyl acetate extract of Knema pachycarpa fruit
}

\author{
To Hai Tung ${ }^{1}$, Cao Thi Hue ${ }^{1}$, Tran Huu Giap ${ }^{1,2}$, Ha Thi Thoa ${ }^{1}$, Nguyen Anh Dung ${ }^{1}$, \\ Nguyen Thi Minh Hang ${ }^{1,2}$, Nguyen Van Hung ${ }^{1,2}$, Le Nguyen Thanh ${ }^{1,2, *}$ \\ ${ }^{1}$ Institute of Marine Biochemistry, Vietnam Academy of Science and Technology (VAST) \\ ${ }^{2}$ Graduate University of Science and Technology, VAST
}

Received 28 September 2016; Accepted for publication 28 August 2017

\begin{abstract}
Knema is a genus of tropical evergreen trees of the family Myristicaceae found in South East Asian countries such as Vietnam, Thailand, and Malaysia. In this paper, four lignans, (+)-pinoresinol (1),(+) epi-pinoresinol (2), piperitol (3), and pluviatilol (4), were isolated from the ethyl acetate extract of the fruit of Knema pachycarpa, an indigenus tree in Vietnam. The chemical structures were determined by spectroscopic data and comparison with the reported literature. These compounds were isolated from Knema genus for the first time.
\end{abstract}

Keywords. Knema pachycarpa de Wilde, (+)-Pinoresinol, (+)-Epi-pinoresinol, Piperitol, Pluviatilol.

\section{INTRODUCTION}

Knema is a genus of tropical evergreen trees of the family Myristicaceae found in South East Asian countries such as Vietnam, Thailand, and Malaysia. At least 13 species are found in Vietnam, where they are commonly known as "mau cho" referring to the red resin secreted in the bark [1]. Traditionally, Knema species have been used to treat sore, pimples, cancers and skin diseases. The genus Knema contains variety of natural compounds including cardanols, flavonoids, acetophenones, lignans, acylphloroglucinols, acylresorcinols, and anacardic acids [2-4].

Knema pachycarpa de Wilde "Mau cho trai day" is an indigenus tree in Vietnam and the chemical study of this Knema species has not been reported. In this paper, we report the isolation of 4 lignan compounds from the ethyl acetate extract of $K$. pachycarpa fruit including (+)-pinoresinol (1), epipinoresinol (2), piperitol (3), and pluviatilol (4). Their chemical structures were determined by spectroscopic data and comparison with the reported literature.

\section{EXPERIMENTAL}

\subsection{General Experimental Procedures}

The ${ }^{1} \mathrm{H}-\mathrm{NMR}(500 \mathrm{MHz})$ and ${ }^{13} \mathrm{C}-\mathrm{NMR}(125 \mathrm{MHz})$ spectra were recorded by a Bruker AM500 FT-NMR spectrometer using TMS as an internal standard. The electrospray ionization mass spectra (ESI-MS) were obtained on an Agilent 1260 series single quadrupole LC/MS system. Column chromatography (CC) was performed on silica gel (Merck, 230-400 mesh) or Sephadex LH-20. Thin layer chromatography used precoated silica gel plates (Merck $60 \mathrm{~F}_{254}$ ). Compounds were visualized by spraying with Ce-Mo stain.

\subsection{Plant material}

The fruit of Knema pachycarpa de Wilde was collected at A-Luoi, Hue city, Viet Nam, in 2015 and identified by Dr. Nguyen The Cuong, Institute of Ecology and Biological Resources, VAST. A voucher specimen (VN-1527) was deposited at the Institute of Marine Biochemistry, VAST.

\subsection{Extraction and Isolation}

The fruits of $K$. pachycarpa were sliced into small pieces and dried. The material $(380 \mathrm{~g})$ was extracted with $\mathrm{MeOH}$ at room temperature (3 times, 1 day/time). The extracts were combined and evaporated in vacuo and the residue was suspended in $\mathrm{H}_{2} \mathrm{O}$. The suspension was successively partitioned with $n$-hexane and ethyl acetate to give $n$-hexane 
residue (105 g) and ethyl acetate residue ( $2.3 \mathrm{~g})$.

The ethyl acetate residue ( $2.28 \mathrm{~g}$ ) was subjected to column chromatography on silica gel, eluted using gradient solvents with $n$-hexane-ethyl acetate (50:1 to $0: 1, \mathrm{v} / \mathrm{v})$ to afford 5 fractions (E1-E5).

The E2 fraction $(702 \mathrm{mg}$ ) was separated into 3 sub-fractions (E2.1-E2.3) using $\mathrm{CC}$ on Sephadex eluted with $\mathrm{MeOH}$. The E2.2 sub-fraction $(618 \mathrm{mg})$ was chromatographed on silica gel column eluted with $\mathrm{CH}_{2} \mathrm{Cl}_{2} / \mathrm{MeOH} 98 / 2$ (v/v) to give 3 subfractions E2.2.1-E2.2.3. Purification of fraction E2.2.1 (230 mg) with silica gel CC eluted with $n$ hexane-ethyl acetate $85: 15 \quad(\mathrm{v} / \mathrm{v})$ furnished compound 1 (24 mg) and compound $2(11 \mathrm{mg})$. The E1 fraction $(400 \mathrm{mg}$ ) was also fractionated by column chromatography on Sephadex eluted with $\mathrm{MeOH}$ to give 3 sub-fractions (E1.1-E1.3). The E1.2 sub-fraction $(68.5 \mathrm{mg})$ was purified on silica gel column using $n$-hexane-ethyl acetate $85: 15(\mathrm{v} / \mathrm{v})$ to yield compound $\mathbf{3}(16.4 \mathrm{mg}$ ) and compound 4 (2.6 $\mathrm{mg})$.

(+)-Pinoresinol (1): white solid, $[\alpha]_{D}^{25}=+75.0^{\circ}$ $\left(\mathrm{CHCl}_{3}, c=0.06\right), \mathrm{mp}: 115-116{ }^{\circ} \mathrm{C}$. ESI-MS: $\mathrm{m} / z$ $359[\mathrm{M}+\mathrm{H}]^{+}$, molecular formula $\mathrm{C}_{20} \mathrm{H}_{22} \mathrm{O}_{6}(\mathrm{M}=$ 358). ${ }^{1} \mathrm{H}-\mathrm{NMR}$ and ${ }^{13} \mathrm{C}$-NMR data, see table 1.

(+)-Epipinoresinol (2): white solid, $[\alpha]_{\mathrm{D}}^{25}=$ $+113.2^{\circ}\left(\mathrm{CHCl}_{3}, c=0.30\right)$, mp: $133-135{ }^{\circ} \mathrm{C}$. ESIMS: $m / z 359[\mathrm{M}+\mathrm{H}]^{+}$, molecular formula $\mathrm{C}_{20} \mathrm{H}_{22} \mathrm{O}_{6}$ ( $\mathrm{M}=358) .{ }^{1} \mathrm{H}-\mathrm{NMR}$ and ${ }^{13} \mathrm{C}-\mathrm{NMR}$ data, see table 1 .

Piperitol (3): clear oil, $[\alpha]_{D}^{25}=-63.6^{\circ}\left(\mathrm{CHCl}_{3}, c\right.$
$=0.25)$. ESI-MS: $m / z 357[\mathrm{M}+\mathrm{H}]^{+}$, molecular formula $\mathrm{C}_{20} \mathrm{H}_{20} \mathrm{O}_{6}(\mathrm{M}=356)$. ${ }^{1} \mathrm{H}-\mathrm{NMR}$ and ${ }^{13} \mathrm{C}$ NMR data, see table 2.

Pluviatilol (4): white solid, $[\alpha]^{25}{ }_{\mathrm{D}}=+36.6^{\circ}$ $\left(\mathrm{CHCl}_{3}, c=0.3\right)$, mp: $160-161{ }^{\circ} \mathrm{C}$. ESI-MS: $\mathrm{m} / z 357$ $[\mathrm{M}+\mathrm{H}]^{+}$, molecular formula $\mathrm{C}_{20} \mathrm{H}_{20} \mathrm{O}_{6}(\mathrm{M}=356)$. ${ }^{1} \mathrm{H}-\mathrm{NMR}$ and ${ }^{13} \mathrm{C}-\mathrm{NMR}$ data, see table 2 .

\section{RESULTS AND DISCUSSION}

Compound 1 was obtained as a white solid. The ESI-MS showed a molecular ion peak $\mathrm{m} / \mathrm{z} 359$ $[\mathrm{M}+\mathrm{H}]^{+}$, indicating that a molecular formula of $\mathbf{1}$ is $\mathrm{C}_{20} \mathrm{H}_{22} \mathrm{O}_{6}$. In the ${ }^{13} \mathrm{C}$-NMR spectra, there were 10 carbon signals suggesting that structure of $\mathbf{1}$ is symmetric. The ${ }^{1} \mathrm{H}$ NMR spectrum revealed the signals $\mathrm{ABX}$ spin systems in the phenyl ring $\left[\delta_{\mathrm{H}}\right.$ : $6.90(1 \mathrm{H}, \mathrm{d}, J=2.5 \mathrm{~Hz}), 6.87(1 \mathrm{H}, \mathrm{d}, J=8.5 \mathrm{~Hz})$, $6.81(1 \mathrm{H}, \mathrm{dd}, J=2.0 \mathrm{~Hz}, J=8.0 \mathrm{~Hz})]$ with a methoxy and hydroxyl group signals at $\delta_{\mathrm{H}} 3.89$ (s, $3 \mathrm{H})$ and 5.68 (br s, 1H), respectively. In addition, the signals of bis-lignan furan ring were found at $\delta_{\mathrm{H}}$ $4.73(1 \mathrm{H}, \mathrm{d}, 4.5 \mathrm{~Hz}), 4.26(1 \mathrm{H}, \mathrm{dd}, J=9 \mathrm{~Hz} ; J=7$ $\mathrm{Hz}), 3.86(1 \mathrm{H}, \mathrm{dd}, J=9 \mathrm{~Hz} ; J=3.5 \mathrm{~Hz})$ and 3.09 $(1 \mathrm{H}, \mathrm{m})$. The ${ }^{13} \mathrm{C}-\mathrm{NMR}$ showed the signals of aromatic carbons at $\delta_{\mathrm{C}} 146.7$ (C-4'), 145.2 (C-3'), 132.9 (C-1'), 118.9 (C-6'), 114.2 (C-5'), 108.6 (C-2'); a methoxy group at $\delta_{\mathrm{C}} 55.96$ and bis-lignan furan ring at $\delta_{\mathrm{C}} 85.8\left(\mathrm{C}-7,7^{\prime}\right), 54.1\left(\mathrm{C}-8,8^{\prime}\right)$ and 71.6 (C-9,9'). Analytical NMR, MS and optical data indicated that the structure of compound $\mathbf{1}$ is (+)pinoresinol. The NMR data is in good agreement with those in the reported literature [5].

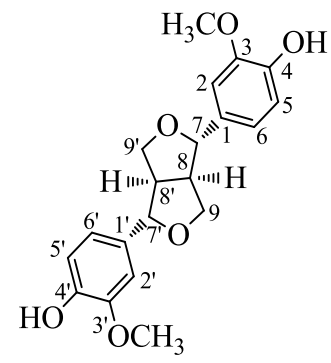

1: (+)-Pinoresinol

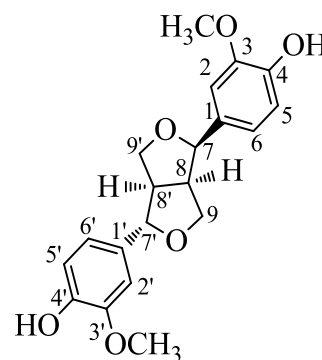

2: (+)-Epipinoresinol

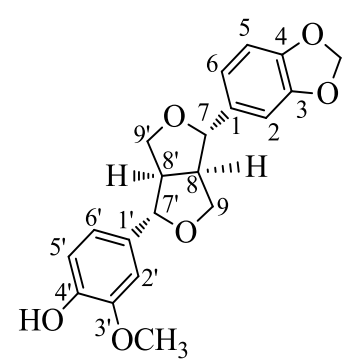

3: Piperitol

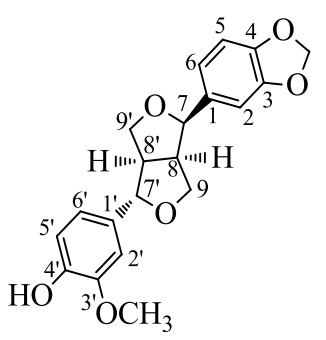

4: Pluviatilol

Fig. 1: Chemical structures of isolated lignans 1-4

Compound 2 was isolated as a white powder, $\mathrm{mp}$ 133-135 ${ }^{\circ} \mathrm{C}$. The ESI-MS (molecular ion peak $\mathrm{m} / \mathrm{z}$ 359) and NMR (20 carbon signals, 22 protons) data indicated that a molecular formula of 2 is $\mathrm{C}_{20} \mathrm{H}_{22} \mathrm{O}_{6}$, the same as compound 1, pinoresinol. The ${ }^{1} \mathrm{H}$ NMR spectrum showed typical signals of two $\mathrm{ABX}$ systems $\left[\delta_{\mathrm{H}} 6.95(\mathrm{~d}, 1 \mathrm{H}, J=1.0 \mathrm{~Hz}), 6.91(\mathrm{~d}, 1 \mathrm{H}, J\right.$
$=1.5 \mathrm{~Hz}), 6.88(\mathrm{~d}, 1 \mathrm{H}, J=8.0 \mathrm{~Hz}), 6.90(\mathrm{~d}, 1 \mathrm{H}, J=$ $8.5 \mathrm{~Hz}), 6.83(\mathrm{dd}, 1 \mathrm{H}, J=8.5 \mathrm{~Hz}, 2.0 \mathrm{~Hz}), 6.77(\mathrm{dd}$, $1 \mathrm{H}, J=8.0 \mathrm{~Hz}, 1.0 \mathrm{~Hz})]$ with two methoxy groups at $3.91(\mathrm{~s}, 3 \mathrm{H}), 3.90(\mathrm{~s}, 3 \mathrm{H})$. The remaining protons signals $\left[\delta_{\mathrm{H}} 4.86(\mathrm{~d}, 1 \mathrm{H}, J=5.5 \mathrm{~Hz}), 4.43(\mathrm{~d}, 1 \mathrm{H}, J=\right.$ $7.0 \mathrm{~Hz}), 4.12(\mathrm{~d}, 1 \mathrm{H}, J=9.5 \mathrm{~Hz}), 3.86-3.83(\mathrm{~m}, 2 \mathrm{H})$, 3.35-3.30 (m, 2H), 2.92-2.88 (m, 1H)] were 
analysed and assigned as epi-furofuran ring using COSY spectrum. The ${ }^{13} \mathrm{C}-\mathrm{NMR}$ and DEPT spectra of 2 showed the signals of 20 carbons including a 12 aromatic carbon signals $\left[\delta_{\mathrm{C}} 146.7,146.4,145.3\right.$, $144.4,133.0,130.3,119.1,118.4,114.2,114.2$, 108.5, and 108.4], 2 methoxy group signals $\left[\delta_{\mathrm{C}}\right.$
56.01 and 55.96] and six carbon signals of epifurofuran ring at $\delta_{\mathrm{C}} 87.75,82.13,71.01,69.69,56.0$, 55.9, 54.4 and 50.1. Therefore, compound 2 was identified as (+) epi-pinoresinol, an isomer of pinoresinol. The NMR data are nearly identical to those of reported (+) epi-pinoresinol [6].

Table 1: ${ }^{1} \mathrm{H}$ and ${ }^{13} \mathrm{C}-\mathrm{NMR}$ data of lignans 1-2 and reference compounds

\begin{tabular}{|c|c|c|c|c|c|c|c|c|}
\hline \multicolumn{5}{|c|}{1} & \multicolumn{4}{|c|}{2} \\
\hline \multirow[b]{2}{*}{$\mathrm{C}$} & \multirow[b]{2}{*}{${ }^{\circledR} \boldsymbol{\delta}_{\mathrm{C}}$} & \multirow[b]{2}{*}{${ }^{\mathbf{a}, \mathbf{b}} \boldsymbol{\delta}_{\mathrm{C}}$} & ${ }^{(@)} \delta_{H}$ & ${ }^{\mathrm{b}, \mathrm{c}} \boldsymbol{\delta}_{\mathrm{H}}$ & \multirow[b]{2}{*}{${ }^{\mathrm{d}} \boldsymbol{\delta}_{\mathrm{C}}$} & \multirow[b]{2}{*}{${ }^{\mathbf{a}, \mathbf{b}} \boldsymbol{\delta}_{\mathrm{C}}$} & ${ }^{\mathbf{b}, \mathbf{c}} \boldsymbol{\delta}_{\mathbf{H}}$ & ${ }^{\mathrm{d}} \delta_{\mathrm{H}}$ \\
\hline & & & (mult., $J=\mathrm{Hz}$ ) & (mult., $J=\mathrm{Hz}$ ) & & & $\begin{array}{l}\text { (mult., } \\
J=\mathrm{Hz} \text { ) }\end{array}$ & $\begin{array}{l}\text { (mult., } \\
J=\mathrm{Hz} \text { ) }\end{array}$ \\
\hline 1 & 132.8 & 132.9 & - & - & 130.8 & 130.3 & - & - \\
\hline 2 & 108.6 & 108.6 & $6.87, \mathrm{~d}(1.6)$ & $6.90, \mathrm{~d}(2.0)$ & 108.9 & 108.4 & $6.95, \mathrm{~d}(1.0)$ & 6.97-6.76, m \\
\hline 3 & 146.5 & 146.7 & - & - & 146.9 & 146.4 & - & - \\
\hline 4 & 145.1 & 145.2 & - & - & 145.1 & 144.4 & - & - \\
\hline 5 & 114.2 & 114.2 & $6.86, \mathrm{~d}(8.0)$ & $6.87, \mathrm{~d}(8.5)$ & 114.7 & 114.2 & $6.88, \mathrm{~d}(8.0)$ & 6.97-6.76, m \\
\hline 6 & 118.9 & 118.9 & 6.79 , dd $(8.0,1.6)$ & $\begin{array}{c}6.81, \text { dd }(8.0, \\
2.0) \\
\end{array}$ & 118.9 & 118.4 & $\begin{array}{c}6.83, \mathrm{dd} \\
(8.5,2.0) \\
\end{array}$ & $6.97-6.76, \mathrm{~m}$ \\
\hline 7 & 85.8 & 85.8 & $4.72, \mathrm{~d}(4.4)$ & $4.73, \mathrm{~d}(4.5)$ & 82.6 & 82.1 & $4.86, \mathrm{~d}(5.5)$ & $4.86, \mathrm{~d}(5.0)$ \\
\hline 8 & 54.2 & 54.1 & $3.08, \mathrm{~m}$ & $3.09, \mathrm{~m}$ & 55.0 & 54.4 & $2.92-288, \mathrm{~m}$ & 2.94-287, m \\
\hline $9 \mathrm{a}$ & \multirow{2}{*}{71.6} & \multirow{2}{*}{71.6} & $\begin{array}{c}4.23 \mathrm{dd} \\
(8.8,6.8)\end{array}$ & $\begin{array}{l}4.26, \mathrm{dd} \\
(9.0,7.0)\end{array}$ & \multirow{2}{*}{70.2} & \multirow{2}{*}{69.6} & $3.86-3.83, \mathrm{~m}$ & $3.89-3.80, \mathrm{~m}$ \\
\hline $9 b$ & & & $3.88, \mathrm{dd}(8.8,3.6)$ & $\begin{array}{c}3.86, \text { dd }(9.0 \\
3.5)\end{array}$ & & & $3.35-3.30, \mathrm{~m}$ & 3.37-3.23, m \\
\hline $1^{\prime}$ & 132.8 & 132.9 & - & - & 133.5 & 133.0 & - & - \\
\hline 2 ' & 108.6 & 108.6 & $6.87, \mathrm{~d}(1.6)$ & $6.90, \mathrm{~s}$ & 109.0 & 108.5 & $6.91, \mathrm{~d}(1.5)$ & 6.97-6.76, m \\
\hline $3^{\prime}$ & 146.5 & 146.7 & - & - & 147.2 & 146.7 & - & - \\
\hline 4 ' & 145.1 & 145.2 & - & - & 145.8 & 145.3 & - & - \\
\hline 5 & 114.2 & 114.2 & $6.86, \mathrm{~d}(8.0)$ & $6.87, \mathrm{~d}(8.5)$ & 114.7 & 114.2 & $6.90, \mathrm{~d}(8.5)$ & 6.97-6.76, m \\
\hline 6 ' & 118.9 & 118.9 & 6.79 , dd $(8.0,1.6)$ & $\begin{array}{c}\text { 6.81, dd (8.0, } \\
2.0)\end{array}$ & 119.6 & 119.1 & $\begin{array}{l}6.77, \mathrm{dd} \\
(8.0,1.0)\end{array}$ & 6.97-6.76, m \\
\hline 7 & 85.8 & 85.8 & $4.72, \mathrm{~d}(4.4)$ & $4.73, \mathrm{~d}(4.5)$ & 88.2 & 87.7 & $4.43, \mathrm{~d}(7.0)$ & $4.44, \mathrm{~d}(7.0)$ \\
\hline 8 ' & 54.2 & 54.1 & $3.08, \mathrm{~m}$ & $3.09, \mathrm{~m}$ & 50.6 & 50.1 & $3.35-3.30, \mathrm{~m}$ & $3.37-3.23, \mathrm{~m}$ \\
\hline $9 a^{\prime}$ & \multirow[b]{2}{*}{71.6} & \multirow[b]{2}{*}{71.6} & $\begin{array}{c}4.23 \mathrm{dd} \\
(8.8,6.8) \\
\end{array}$ & $\begin{array}{l}4.26, \mathrm{dd} \\
(9.0,7.0)\end{array}$ & \multirow[b]{2}{*}{71.5} & \multirow[b]{2}{*}{71.0} & $4.12, \mathrm{~d}(9.5)$ & $4.12, \mathrm{~d}(9.3)$ \\
\hline $9 b^{\prime}$ & & & 3.88 , dd $(8.8,3.6)$ & $\begin{array}{c}\text { 3.86, dd (9.0, } \\
3.5)\end{array}$ & & & $3.86-3.83, \mathrm{~m}$ & 3.89-3.80, m \\
\hline $3-\mathrm{OCH}_{3}$ & 55.9 & 55.9 & 3.83 & 3.89 & 56.5 & 56.0 & $3.91, \mathrm{~s}$ & $3.91, \mathrm{~s}$ \\
\hline $3{ }^{\prime}-\mathrm{OCH}_{3}$ & 55.9 & 55.9 & 3.83 & 3.89 & 56.4 & 55.9 & $3.90, \mathrm{~s}$ & $3.89, \mathrm{~s}$ \\
\hline
\end{tabular}

${ }^{\mathrm{a}} 125 \mathrm{MHz},{ }^{\mathrm{b}} \mathrm{CDCl}_{3},{ }^{\mathrm{c}} 500 \mathrm{MHz}$, @: (+)-Pinoresinol [5], d: (+)-Epi-pinoresinol [6].

Compound $\mathbf{3}$ was isolated as an oil. The NMR features indicate that the structure of $\mathbf{3}$ is also a lignan. The ${ }^{1} \mathrm{H}$ NMR spectrum showed 6 signals of two $\mathrm{ABX}$ spin systems in the aromatic region $\left[\delta_{\mathrm{H}}\right.$ : 6.87-6.89 $(2 \mathrm{H}, \mathrm{m}), 6.85(1 \mathrm{H}, \mathrm{d}, J=1.5 \mathrm{~Hz}), 6.79$ $6.82(2 \mathrm{H}, \mathrm{m})$ and $6.77(1 \mathrm{H}, \mathrm{s})]$, with a methylene dioxide, hydroxyl and methoxy group signals at $\delta_{\mathrm{H}}$ $5.94(2 \mathrm{H}, \mathrm{s}), 5.62(1 \mathrm{H}, \mathrm{s})$ and $3.90(3 \mathrm{H}, \mathrm{s})$, respectively. The signals of bis-lignan furan $\operatorname{ring}\left[\delta_{\mathrm{H}}\right.$ $4.73(\mathrm{dd}, 4.5,2.0,2 \mathrm{H}), 4.26-4.21(\mathrm{dd}, 9.0,6.5,2 \mathrm{H})$, 3.89-3.85 (dd, 9.0, 4.0, 2H) and 3.11-3.03 (2H, m)] are similar to those of pinoresinol. The ${ }^{13} \mathrm{C}-\mathrm{NMR}$ 
showed 20 carbon including 12 signals of aromatic carbons, methylene dioxide group at $\delta_{\mathrm{C}} 101.07$, a methoxy group at $\delta_{\mathrm{C}} 55.96$ and bis-lignan furan ring at $\delta_{\mathrm{C}} 85.87,85.83,71.72,71.68,54.33$ and 54.18. The ESI-MS showed a molecular ion peak $\mathrm{m} / \mathrm{z} 357$
$[\mathrm{M}+\mathrm{H}]^{+}$, indicating that a molecular formula of $\mathbf{3}$ is $\mathrm{C}_{20} \mathrm{H}_{20} \mathrm{O}_{6}$. On the basis of the above spectral evidences, compound $\mathbf{3}$ is determined as piperitol. The analytical NMR data of $\mathbf{3}$ are in accordance with those published $[7,8]$.

Table 2: ${ }^{1} \mathrm{H}$ and ${ }^{13} \mathrm{C}-\mathrm{NMR}$ data of lignans 3-4 and reference compounds

\begin{tabular}{|c|c|c|c|c|c|c|}
\hline \multicolumn{5}{|c|}{3} & \multicolumn{2}{|r|}{4} \\
\hline \multirow{2}{*}{$\mathrm{C}$} & \multirow{2}{*}{${ }^{\#} \boldsymbol{\delta}_{\mathrm{C}}$} & \multirow{2}{*}{${ }^{\mathbf{a}, \mathbf{b}} \boldsymbol{\delta}_{\mathrm{C}}$} & 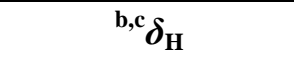 & ${ }^{*} \delta_{\mathrm{H}}$ & 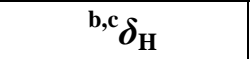 & ${ }^{\&} \delta_{\mathrm{H}}$ \\
\hline & & & (mult., $J=\mathrm{Hz}$ ) & (mult., $J=\mathrm{Hz}$ ) & (mult., $J=\mathrm{Hz}$ ) & (mult., $J=\mathrm{Hz}$ ) \\
\hline 1 & 135.1 & 135.1 & - & - & - & - \\
\hline 2 & 106.5 & 106.5 & $6.77-6.89, \mathrm{~m}$ & $6.76-6.93, \mathrm{~m}$ & $6.77-6.94, \mathrm{~m}$ & $6.81-6.89, \mathrm{~m}$ \\
\hline 3 & 148.0 & 147.9 & - & - & - & - \\
\hline 4 & 146.8 & 146.7 & - & - & - & - \\
\hline 5 & 108.2 & 108.1 & $6.77-6.89, \mathrm{~m}$ & $6.76-6.93, \mathrm{~m}$ & 6.77-6.94, m & $6.81-6.89, \mathrm{~m}$ \\
\hline 6 & 119.3 & 119.3 & $6.77-6.89, \mathrm{~m}$ & $6.76-6.93, \mathrm{~m}$ & $6.77-6.94, \mathrm{~m}$ & $6.81-6.89, \mathrm{~m}$ \\
\hline 7 & 85.9 & 85.8 & $4.73, \mathrm{dd}(4.5,2.0)$ & $4.72, \mathrm{~d}(4.5)$ & $4.85, \mathrm{~d}(4.5)$ & $4.86, \mathrm{~d}(6.0)$ \\
\hline 8 & 54.3 & 54.3 & $3.03-3.11, \mathrm{~m}$ & $2.85-3.25, \mathrm{~m}$ & $3.31, \mathrm{~m}$ & $3.32, \mathrm{~m}$ \\
\hline $9 \mathrm{a}$ & \multirow{2}{*}{71.7} & \multirow{2}{*}{71.7} & $4.26, \mathrm{dd}(9.0,6.5)$ & 4.26 , dd $(9.0,6.5)$ & $3.84, \mathrm{~m}$ & $3.85, \mathrm{~m}$ \\
\hline $9 b$ & & & $3.89, \mathrm{dd}(9.0,4.0)$ & 3.85 , dd $(9.0,3.5)$ & $3.30, \mathrm{~m}$ & $3.32, \mathrm{~m}$ \\
\hline 1 ' & 132.9 & 132.9 & - & - & - & - \\
\hline 2 ' & 108.7 & 108.6 & $6.77-6.89, \mathrm{~m}$ & $6.76-6.93, \mathrm{~m}$ & $6.77-6.94, \mathrm{~m}$ & $6.81-6.89, \mathrm{~m}$ \\
\hline $3^{\prime}$ & 147.1 & 147.1 & - & - & - & - \\
\hline 4, & 145.3 & 145.2 & - & - & - & - \\
\hline 5, & 114.4 & 114.3 & $6.77-6.89, \mathrm{~m}$ & $6.76-6.93, \mathrm{~m}$ & $6.77-6.94, \mathrm{~m}$ & $6.81-6.89, \mathrm{~m}$ \\
\hline 6 & 119.0 & 119.0 & $6.77-6.89, \mathrm{~m}$ & $6.76-6.93, \mathrm{~m}$ & $6.77-6.94, \mathrm{~m}$ & $6.81-6.89, \mathrm{~m}$ \\
\hline 7 , & 85.9 & 85.8 & $4.73, \mathrm{dd}(4.5,2.0)$ & $4.72, \mathrm{~d}(4.5)$ & $4.43, \mathrm{~d}(7.0)$ & $4.42, \mathrm{~d}(7.5)$ \\
\hline $8^{\prime}$ & 54.2 & 54.1 & $3.03-3.11, \mathrm{~m}$ & $2.85-3.25, \mathrm{~m}$ & $2.88, \mathrm{~m}$ & $2.91, \mathrm{~m}$ \\
\hline $9 a^{\prime}$ & \multirow{2}{*}{71.7} & \multirow{2}{*}{71.6} & $4.26, \mathrm{dd}(9.0,6.5)$ & $4.26, \mathrm{dd}(9.0,6.5)$ & $4.11, \mathrm{~d}(9.5)$ & $4.13, \mathrm{dd}(9.5,1.0)$ \\
\hline $9 b^{\prime}$ & & & 3.89 , dd $(9.0,4.0)$ & $3.85, \mathrm{dd}(9.0,3.5)$ & $3.86, \mathrm{~m}$ & $3.85, \mathrm{dd}(9.5,6.5)$ \\
\hline $3^{\prime}-\mathrm{OCH}_{3}$ & 56.0 & 55.9 & $3.90, \mathrm{~s}$ & $3.90, \mathrm{~s}$ & $3.91, \mathrm{~s}$ & $3.91, \mathrm{~s}$ \\
\hline$-\mathrm{OH}$ & & & 5.62 , br s & 5.75 , br s & 5.76 , br s & \\
\hline$-\mathrm{OCH}_{2} \mathrm{O}-$ & 101.1 & 101.0 & $5.94, \mathrm{~s}$ & $5.96, \mathrm{~s}$ & $5.94, \mathrm{~s}$ & $5.97, \mathrm{~s}$ \\
\hline
\end{tabular}

${ }^{\mathrm{a}} 125 \mathrm{MHz},{ }^{\mathrm{b}} \mathrm{CDCl}_{3},{ }^{\mathrm{c}} 500 \mathrm{MHz},{ }^{*} \delta_{\mathrm{H}}$ : Piperitol [7], ${ }^{\#} \delta_{\mathrm{C}}$ : Piperitol [8], ${ }^{\&} \delta_{\mathrm{H}}$ : Pluviatilol [9].

Compound 4 was obtained as a white solid, $\mathrm{mp}$ $160-161{ }^{\circ} \mathrm{C}$. In the NMR spectrum, the proton signals of aromatic ring are similar to those of compound 3 (piperitol) with 6 proton signals in the aromatic region, a methylene dioxide, a hydroxyl and a methoxy group at $\delta_{\mathrm{H}} 5.94(2 \mathrm{H}, \mathrm{s}), 5.76(1 \mathrm{H}, \mathrm{s})$ and $3.91(3 \mathrm{H}, \mathrm{s})$. However, the remaining protons signals are similar to those of compound 2, epipinoresinol with 8 protons at $\delta_{\mathrm{H}} 4.85(1 \mathrm{H}, \mathrm{d}, J=4.5$ $\mathrm{Hz}), 4.43(1 \mathrm{H}, \mathrm{d}, J=7.0 \mathrm{~Hz}), 4.11(1 \mathrm{H}, \mathrm{d}, J=9.5$
$\mathrm{Hz}), 3.86-3.84(2 \mathrm{H}, \mathrm{m}), 3.31-3.30(2 \mathrm{H}, \mathrm{m}), 2.88$ $(1 \mathrm{H}, \mathrm{m})$. Therefore, compound $\mathbf{4}$ was identified as pluviatilol, an isomer of piperitol. The ${ }^{1} \mathrm{H}-\mathrm{NMR}$ data are in good agreement with the reported literature [9].

\section{CONCLUSION}

A phytochemical investigation of the ethyl acetate extract of the fruit of $K$. pachycarpa led to the 
isolation of four lignans including (+)-pinoresinol (1), (+) epi-pinoresinol (2), piperitol (3), and pluviatilol (4). Their chemical structures were elucidated by spectroscopic NMR and MS data. These lignans were isolated from Knema genus for the first time.

Acknowledgments. The authors gratefully acknowledge the supports of Institute of Marine Biochemistry under grant number HSB16-CSO4.

\section{REFERENCES}

1. Pham Hoang Ho. An illustrated flora of Vietnam. 282-285, Youth Publisher (1999).

2. M. N. Akhtar, K. W. Lam, F. Abas, Maulidiani, A. Ahmad, S. A. A. Shah, Atta-ur-Rahman, M. I. Choudhary, N. H. Lajis. New class of acetylcholinesterase inhibitors from the stem bark of Knema laurina and their structural insights. Bioorg. Med. Chem. Lett., 21, 4097-4103 (2011).

3. N. Rangkaew, R. Suttisri, M. Moriyasu, K. Kawanishi. A new acyclic diterpene and bioactive compounds from Knema glauca, Arch. Pharm. Res.,
32, 685-692 (2009).

4. M. J. T. G. Gonzalez, M. M. M.Pinto, A. Kijjoa, C. Anantachoke, W. Herz. Stilbenes and other constituents of Knema austrosiamensis. Phytochemistry, 32, 433-438 (1993).

5. H. P. Ji, W. Y. Seung, G. C. Jin, Y. L. Dae, S. K. Yong, and I. B. Nam. Lignans from silkworm droppings and their promotional activities on heme oxygenase-1 (HO-1), J. Korean Soc. Appl. Biol. Chem., 53(6), 734-739 (2010).

6. A. S. Nigel, C. D. B. Richard and B. Gordon. A versatile stereoselective synthesis of endo,exofurofuranones: Application to the enantioselective synthesis of furofuran lignans, J. Org. Chem., 69 , 122-129 (2004).

7. Fumiko, Y. Shoji, K. Kimiko, N. Genichiro, O. Hikaru, N. Istuo. Studies on Xanthoxylum spp. II. Constituents of the bark of Xanthoxylum piperitum DC., Chem. Pharm. Bull., 22, 2650-2655 (1974).

8. T. Hitoshi, N. Takeshi, I. Kazuhiko, and I. Kazuo. A lignan from Actinodaphne longifolia, Phytochemistry, 28(3), 952-954 (1989).

9. S. Z. Choi, M. C. Yang, S. U. Choi and K. R. Lee. Cytotoxic terpenes and lignans from the roots of Ainsliaea acerifolia, Arch. Pham. Res., 29(3), 203208 (2006).

\section{Corresponding author: Le Nguyen Thanh}

Institute of Marine Biochemistry

Vietnam Academy of Science and Technology

No. 18, Hoang Quoc Viet Road, Cau Giay Dist., Hanoi

E-mail: lethanh@imbc.vast.vn; Telephone: 0983882573. 